\title{
Neuartige, differentiell gespeiste Transmissionsleitung auf Silicium
}

\author{
S. El Rai \\ ATMEL Duisburg GmbH, Bismarckstr. 120, 47057 Duisburg, Germany
}

Zusammenfassung. Die elektrische Länge von technisch relevanten TLs liegt zwischen $\lambda / 8$ und $\lambda$. Klassische TLs weisen jedoch kaum praktikabele Ausmaße in Ausbreitungsrichtung auf, (um sie in integrierte Schaltungen zu verwenden). Weiterhin besitzen die klassischen TLs hohe elektrische Dämpfungen, sobald ein hoher Wellenwiderstand angestrebt wird.

Im vorliegender Artikel wird gezeigt, wie durch geometrisches Verändern der Leitungskonfiguration und Einfügen von MIM Kapazitäten herkömmliche, differentiell gespeisten Transmissionsleitungen gleichzeitig deren Leitungsabmessungen und deren Dämpfung bei gleicher elektrischer Länge bedeutend verringert werden kann.

\section{Einleitung}

Zur Übertragung und Verarbeitung von hochfrequenten Signalen in differentielle Schaltungen werden sehr oft symmetrisch aufgebaute, differentiell gespeiste Transmissionsleitungen (DTL) verwendet. Derartige TLs sind für das "Microwave Monolithic Integrated Circuit" (MMIC) Schaltungsdesign von großer Bedeutung.

Die elektrische Länge von technisch relevanten TLs liegt zwischen $\lambda / 8$ und $\lambda$. Klassische TLs weisen jedoch kaum praktikabele Ausmaße in Ausbreitungsrichtung auf. Weiterhin besitzen die klassischen TLs hohe elektrische Dämpfungen, wenn ein hoher Wellenwiderstand angestrebt wird.

Die differentiell gespeisten Leitungen können durch ihre Welleneigenschaften charakteristische Impedanz $\underline{Z}$ und Ausbreitungskonstante $\gamma \rightarrow Q$ (Güte) charakterisiert werden. Während im Bild 1a das Symbol einer klassischen Leitung zu sehen ist, zeigt Bild 1b die Möglichkeiten, die ein Entwickler hat die Eigenschaften dieser Leitung zu beeinflussen. Eine Veränderung der Leitungseigenschaften kann

Correspondence to: S. El Rai (selrai@ddc.atmel.com)

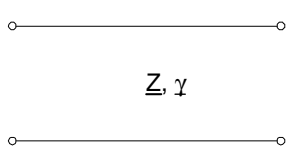

(a)

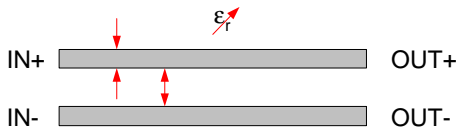

(b)
Abbildung 1. (a) Ersatzschaltbild einer Leitung und (b) Möglichkeiten zur Beeinflussung der Welleneigenschaften derselben: über Veränderung des Leiterquerschnitts, Leiterabstands und der Dielektrizitätskonstante.

durch Veränderung des Leitungsquerschnitts, des Abstands der beiden Leiter, des Abstands der Leiter zu Masse oder der Dielektrizitätskonstante des umgebenden Dielektrikums geschehen. Alle diese Maßnahmen verändern die lateralen $\mathrm{Ab}$ messungen des Leiters nicht entscheidend. Auf Grund der großen Leiterabmessungen finden Leitungen in MMIC bei Frequenzen um $10 \mathrm{GHz}$ so gut wie keine Anwendung.

\section{Design von stark verkürzten differentiellen Leitungen}

Nun gilt es die Abmessungen von herkömmlichen symmetrischen, differentiell gespeisten TLs (Bild 2a) soweit zu verkleinern, dass sie im MMIC Design Anwendung finden können unter der Voraussetzung, das sie homogene Übertragungseigenschaften zeigen. Bekannt geworden sind so genannte Mäander-Leitungen (Bild 2b). Neben dem Vorteil der Verringerung der absoluten Länge bzw. Abmessung der gestreckten Leitung (durch Schleifenlegung) - auf Kosten einer größeren Weite der gesamten Struktur - gesellen sich aber eine Reihe von Nachteilen.

Eine differentiell $\left(180^{\circ}\right.$ Phasenunterschied) gespeiste TL weist auf beiden Leitern einen entgegengesetzten Stromfluss auf. Dementsprechend wird um jeden Leiter ein magnetisches Feld generiert. Die sich nah gegenüber befindlichen 


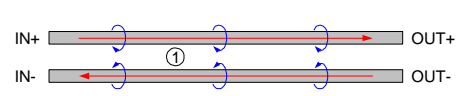

(a)

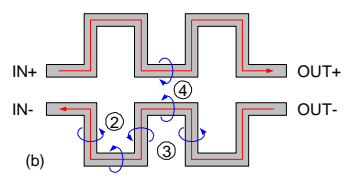

Abbildung 2. Strom- und magnetische Feldverteilung in einer symmetrischen, differentiell gespeisten Leitung: (a) als gestreckter Leiter und (b) als Mäander-Leiter.

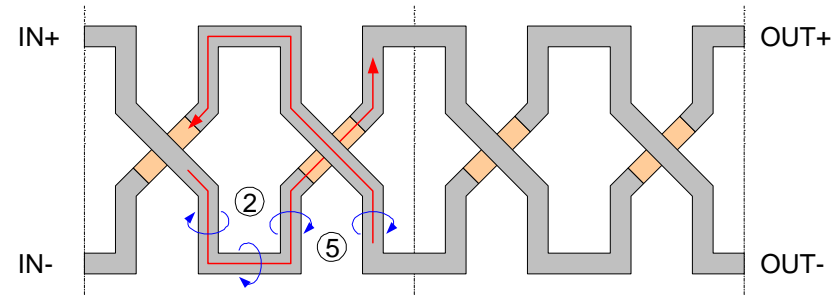

Abbildung 3. Verbesserte Leitungsführung: durch gegenseitiges Kreuzen der Leiter der symmetrischen Leitung.

Leiterstücke haben eine negative magnetische Kopplung, das führt zu erhöhten Verlusten (Naheffekt) (El Rai, 2003). Betrachtet man nun im Bild $2 b$ eine Mäander-Leitung, so ergeben sich magnetische Verluste nicht nur zwischen den Leiterabschnitten (4), sondern auch noch zwischen Abschnitten des selben Leiters, die die Gebiete (2) und (3) begrenzen. Daher wird die elektrische Länge der Leiter aus Bild $2 b$ nicht der geometrischen Länge der sich aus der einfachen Addition der einzelnen Leiterstücklänge ergibt, entsprechen. Ursache hierfür ist die negative magnetische Kopplung. Aus diesen Grund (und anderen wie z.B. Eck-Effekte) weist die Mäander-Leitung bei gleicher elektrische Länge höhere Verluste auf als eine gestreckte TL.

Für eine einfache feldtheoretischen Berechnung unterteilt man die Leitung in entsprechende - immer wiederkehrende - Abschnitte. Wir nennen diese Abschnitte Periodische Struktur (PS). Nähere Einzelheiten sind in El Rai und Tempel (2006) zu finden. In den Bildern 3-5 sind jeweils Leitungen, bestehend aus zwei PS, dargestellt.

Durch geschickte Leitungsführung gelingt es, die magnetische Kopplung manche Leitungsstücke positiv zu gestalten und somit den Aufbau des magnetischen Feldes zu verstärken. Dazu sollten die Gebiete eliminiert werden, in denen eine negative Überlagerung der magnetischen Felder stattfindet. So wird der Strompfad - im Unterschied zu Bild $2 b-$ nun in gekreuzter Form gemäß Bild 3 geführt. Man erkennt den Wegfall der Gebiete (4) und der Gebiete (3) jetzt ersetzt durch Gebiete (5). Lediglich die Gebiete (2) sind erhalten geblieben. Die Leitungsstuicke, die das Gebiet (5) abgrenzen, habe jetzt eine positive magnetische Kopplung (da die Ströme gleiche Richtung haben); das führt zu einer Erhöhung der elektrische Länge bei gleicher geometrischen Länge und somit nahezu gleich bleibenden Verlusten. Die Gesamtverluste pro elektrischer Länge sind nun aber gerin-

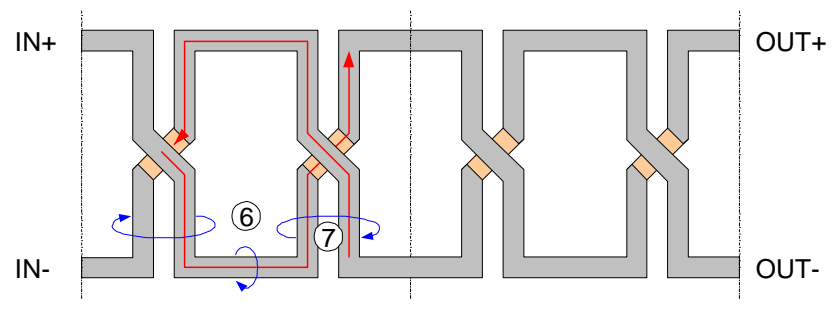

Abbildung 4. Optimierte Leitungsführung: durch gegenseitiges Kreuzen der Leiter der symmetrischen Leitung und durch Abstandsvergrößerung in den Gebieten mit negativer magnetischer Kopplung und Abstandsverringerung in den Gebieten mit positiver magnetischer Kopplung.

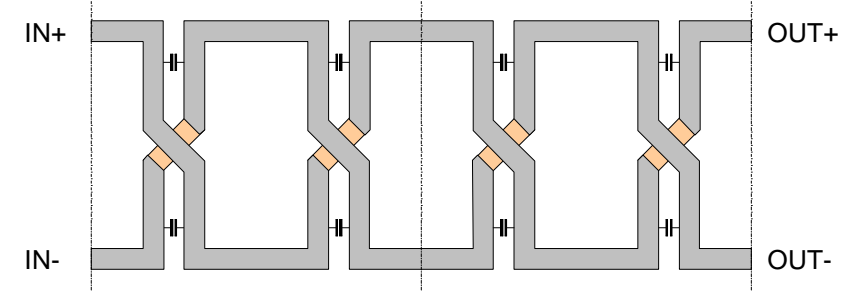

Abbildung 5. Optimierte Leitungsführung und Hinzufügen von Kapazitäten in Gebieten mit positiver magnetischer Kopplung.

ger als bei der traditionellen TL. Nachteil: man braucht mindestens zwei Metalllagen zur Realisierung.

Eine Optimierung der Geometrie in Bild 3 kann dahingehend erfolgen, dass Leitungen, die das Gebiete (5) abgrenzen und positive magnetischen Kopplung haben, näher zu einander angeordnet werden und Leitungen, die das Gebiete (2) abgrenzen und negative magnetische Kopplung besitzen weiter auseinander. Die Gebiete (2) aus Bild 3 werden zu Gebieten (6) in Bild 4 und genauso wird aus (5) nun (7). Durch eine geeignete Wahl der Leiterbahnbreite, den Leiterabständen und der Anzahl der PS lässt sich eine optimale Geometrie für eine bestimmte Fläche mit bestimmter Dämpfung finden - und umgekehrt.

Die Leitungsstücke (mit gleicher Stromrichtung), die das Gebiet (7) abgrenzen werden von magnetischen Feldlinien umrandet, deshalb erscheint es günstig, dieses Gebiet für die Unterbringung von zusätzlichen Bauelementen zu benutzen. Durch Unterbringung von Kapazitäten entlang der gemeinsam verlaufenden Leitungsabschnitte in (7) gelingt es den Kapazitätsbelag der TL entscheidend zu ändern (Hayden und Rebeiz, 2000; Eleftheriades et al., 2002). Man erhält dadurch noch höhere Verkürzungsfaktoren. Verwendet man variable Kapazitäten - z.B. Varaktoren (Nagra et al., 1999; Ellinger et al., 2001; Kim et al., 2005) - kann man die Eigenschaften der Leitung (Impedanz und elektrische Länge) nachträglich elektrisch verändern bzw. abstimmen.

In Bild 5 wird schließlich der nahezu magnetisch feldfreie Raum ((7) in Bild 4) ausgenutzt, indem zusätzliche Kapazitäten im elektrischen Feld untergebracht werden. Hier 


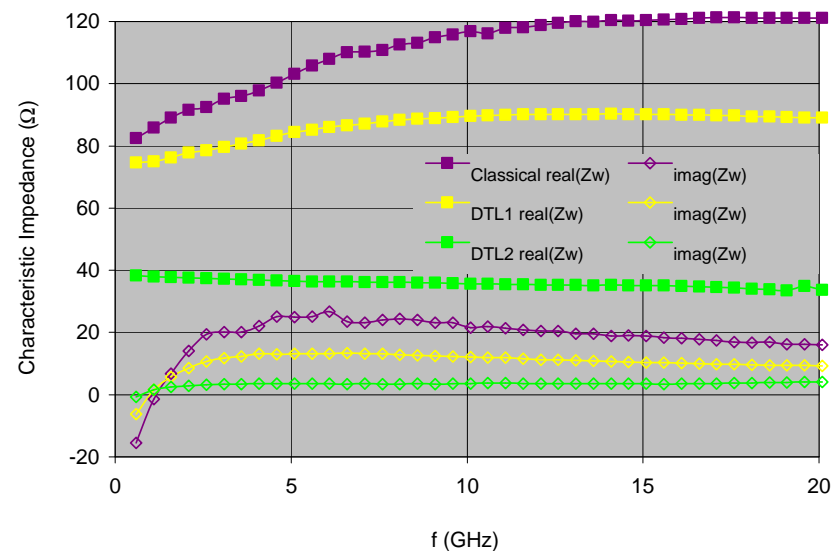

Abbildung 6. Real- und Imaginärteil des Wellenwiderstandes der drei Leitungen.

erniedrigt sich die Impedanz bei gleichzeitiger Erhöhung der Güte.

\section{Simulation und Herstellung der Leitung}

Wegen der starken elektro-magnetischen Verkopplung der einzelnen Leitungsstrukturen untereinander ist die Simulation der Leitungseigenschaften sehr aufwändig. Für die Berechnung wird der 3-D-EM Simulator "Empire" (3-D-FDTD Simulator EMPIRE Version 4.1, IMST GmbH, KampLintfort, Germany, 2004), der auf der Methode der Finiten Differenzen im Zeitbereich (FDTD) basiert.

Als Basis - sowohl für die Simulation als auch die eigentliche Herstellung der Leitungen - diente ATMEL's $0.35 \mu \mathrm{m}$ SiGe BiCMOS Prozess (AT46000 Rev 0.7 SiGe BiCMOS Technology, ATMEL Corp., Colorado Springs, CO, USA, 2004., 2004). Benutzt wurde eine Variante mit drei Metall Ebenen sowie eine zusätzliche Spulenmetall Ebene auf einem 19- $\Omega \mathrm{cm}$ Si-Substrat.

\section{Design und Ergebnisse}

Es werden drei Leitungen evaluiert. Dabei dient Leitung 1 (Classical) als Vergleichsgrundlage; sie ist als klassische Mäanderleitung (s.a. Bild 2b) aufgebaut. Leitung 2 (DTL1) stellt die eine neue Variante (gemäß Bild 4) dar. Leitung 3 (DTL2) ist gemäß Bild 5 - mit zusätzlichen Metall-IsolatorMetall (MIM) Kapazitäten - aufgebaut.

Die nachfolgenden Ergebnisse sind für die Länge einer PS dargestellt. Die Ergebnisse für die komplette Leitung sind für die Leitungsimpedanz und Q-Faktor identisch dazu und für die Phasenverschiebung das Produkt aus derselben und der Anzahl der PS (El Rai und Tempel, 2006).

Bild 6 zeigt den Verlauf für den Real- und Imaginärteil des Wellenwiderstandes. Während die klassische Mäanderleitung den höchsten Wert für den Realteil erzielt, sinkt der

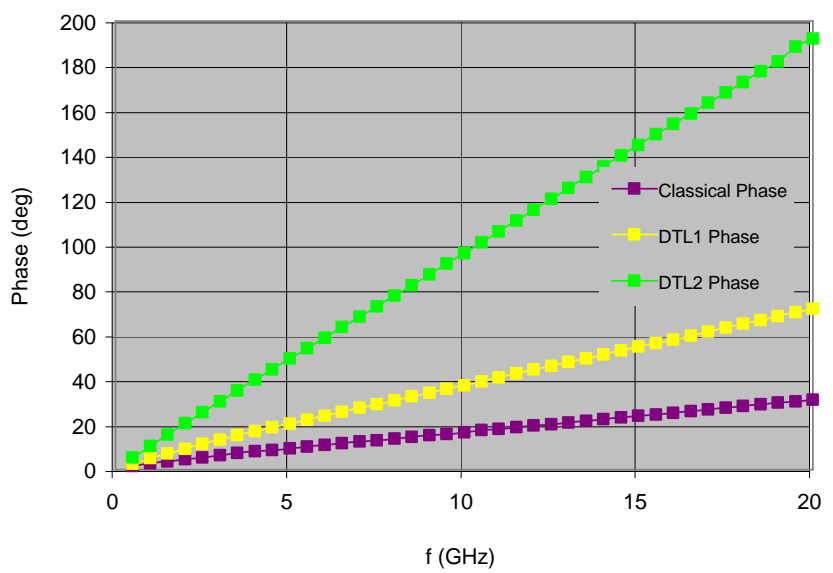

Abbildung 7. Elektrische Länge bzw. Phasenverschiebung über der Frequenz der drei Leitungen.

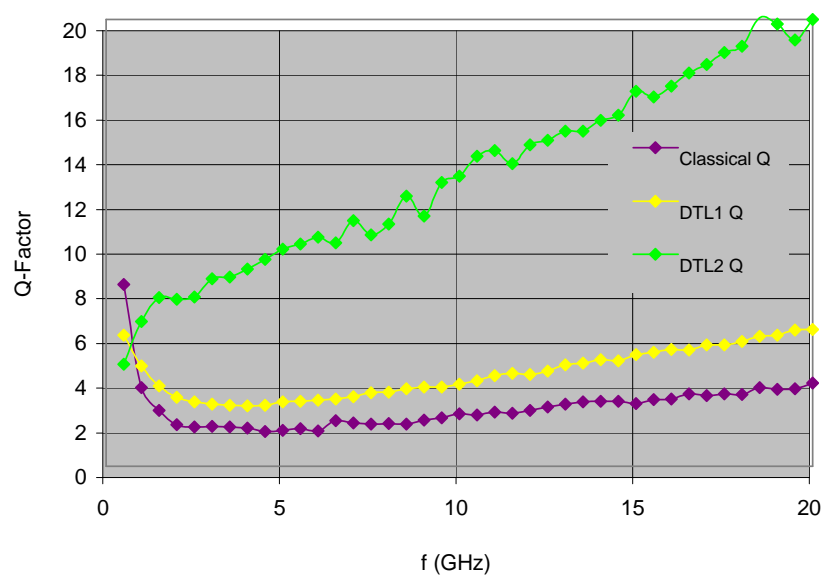

Abbildung 8. Q-Faktor über der Frequenz der drei Leitungen.

Wert für die neuartigen DTLs durch höhere kapazitive Belastung der Leitung immer weiter ab; am stärksten für DTL2. Der Imaginärteil für die klassische Mäanderleitung ist am höchsten und sinkt für die DTL2 Leitung auf einen verschwindenden Anteil ab.

Bild 7 zeigt den Verlauf der Phasenlage bzw. der elektrischen Länge über die Frequenz. Während die klassische Mäanderleitung lediglich einen relativ kleinen Betrag von ca. $17^{\circ}$ bei $10 \mathrm{GHz}$ zeigt erbringt die DTL1 Leitung gut das doppelte von ca. $36^{\circ}$, die DTL2 Leitung erbringt dagegen fast den 6-fachen Wert von $96^{\circ}$. Mit anderen Worten: die letztgenannte Leitung verkürzt sich gegenüber der Mäanderleitung auf 1/6 ihrer originalen Länge!

Bild 8 zeigt den Verlauf des Q-Faktors über der Frequenz. Den niedrigsten hat erwartungsgemäß die klassische Mäanderleitung mit 2.33 bei $10 \mathrm{GHz}$. Die DTL1 Leitung weist ein Q von 3.7 auf, während die DTL2 Leitung ein Q von 13 zeigt, nahezu versechsfacht.

Der Vergleich mit Messungen erfolgt zu einem späteren 
Zeitpunkt, da die Teststrukturen sich noch in der Präparation befinden.

\section{Zusammenfassung}

Es wurde eine neue Methode zur Verkürzung von differentiellen Transmissionsleitungen entwickelt. Dabei wurde gezeigt, dass durch geeignete Anordnung der einzelnen Leiterstücken eine Vergrößerung der magnetischen Kopplung zwischen den Leiterstücken und damit eine Erhöhung der elektrischen Länge und des Q-Faktors, bei gleichen äußeren Leitungsabmessungen, erfolgt. Zusätzlich bietet dieser Anordnung geeignete Stellen zur Unterbringen von MIM Kapazitäten (zwei verschiedene Metallebenen) bzw., von anderen Bauelementen - ohne Verletzung der Symmetrie der Leitungen, welches zu einer weiteren Erhöhung des kapazitiven Belages zwischen den beiden Leitungen führt und somit wiederum zu einer weiteren Erhöhung der elektrischen Länge.

\section{Referenzen}

El Rai, S.: Simulation und Optimierung von 3D Leiterstrukturen für den Einsatz in Hochfrequenz ASICs", Diplomarbeit, Univ. Duisburg-Essen, FG Optoelektronik, 2003.
El Rai, S. and Tempel, R.: Differential Transmission Lines on Silicon Based on Periodic Photonic Bandgap Structures, RWS-2006, 17.-19. Januar 2006, unter Veröffentlichung.

Wien, A.: 3D-FDTD Simulator EMPIRE Version 4.1, IMST GmbH, Kamp-Lintfort, Germany, 2004.

Carver, D.: AT46000 Rev 0.7 SiGe BiCMOS Techn., ATMEL Corp., Colorado Springs, CO, USA, 2004.

Hayden, J. S. and Rebeiz, G. M.: Low-Loss Cascadable MEMS Distributed $\mathrm{x}$-Band Phase Shifters, IEEE Microwave and Guided Wave Letters, 10, 142-144, 2000.

Eleftheriades, G. V., Iyer, A. K., and Kremer, P. C: Planar negative refractive index media using Periodically L-C Loaded Transmission Lines, IEEE Trans. On Microwave Theory and Techn., 50, 2702-2712, 2002.

Nagra, A. S., Xu, J., Erker, E., and York, R. A.: Monolithic GaAs Phase Shifter Circuit with Low Insertion Loss and Continous 0$360^{\circ}$ Phase Shift at $20 \mathrm{GHz}$ ", IEEE Microwave and Guided Wave Letters, 9, 31-33, 1999.

Ellinger, F., Vogt, R., and Bächthold, W.: Ultra Compact, Low Loss, Varactor Tuned Phase Shifter MMIC at C-Band, IEEE Microwave and Wireless Comp. Let., 11, 104-105, 2001.

Kim, ., Ho, S.-J., Yen, C.-C., Sun, K.-O., and van der Weide, D. W $\ddot{\iota}$ : Balanced Distributed-Elements Phase Shifter, IEEE Microwave and Wireless Components Letters, 15, 147-149, 2005. 